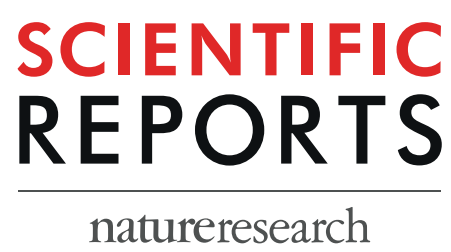

\title{
OPEN Microbiota and metabolome responses in the cecum and serum of broiler chickens fed with plant essential oils or virginiamycin
}

\begin{abstract}
Yan Chen, Jun Wang, LongfeiYu, Tianyue Xu \& Nianhua Zhu*
This study investigated the cecal microbiota and serum metabolite profile of chickens fed with plant essential oils (PEO) or virginiamycin (VIRG) using high-throughput 16S rRNA gene sequencing and untargeted metabolomics approach. The main aim of this work was to explore the biochemical mechanisms involved in the improved growth performance of antibiotics and their alternatives in animal production. The results showed that both PEO and VIRG treatment significantly increased the relative abundance of phyla Bacteroidetes and decreased the abundance of phyla Firmicutes and genus of Lactobacillus in cecal microbiota of chickens. Compared to the control group (CT group), the relative abundance of genus of Alistipes, unclassified Rikenellaceae, Roseburia, and Anaeroplasma was enriched in the PEO group; that of genus Bacteroides, Lachnospiraceae, and unclassified Enterobacteriaceae was enriched in the cecal microbiota of the VIRG group. Untargeted metabolomics analyses revealed that the PEO treatment modified 102 metabolites and 3 KEGG pathways (primary bile acid biosynthesis and phenylalanine metabolism) in the cecal microbiota, and 81 metabolites and relevant KEGG pathways (fructose and mannose metabolism, biosynthesis of unsaturated fatty acids, and linoleic acid.) in the serum of the chicken. Compared to the CT group, VIRG treatment group differed 217 metabolites and 10 KEGG pathways in cecal contents and 142 metabolites and 7 KEGG pathways in serum of chickens. Pearson's correlation analysis showed that phyla Bacteroidetes and genus of Bacteroides, Alistipes, and unclassified Rikenellaceae (in the VIRG and PE group) were positively correlated with many lipid metabolites. However, phyla Firmicutes and genera Lactobacillus (higher in the CT group) were negatively correlated with the lipid and thymine metabolism, and positively correlated with hydroxyisocaproic acid, cytosine, and taurine. This study shows that dietary supplementation with PEO and VIRG altered the composition and metabolism profile of the cecal microbiota, modified the serum metabolism profile.
\end{abstract}

The intestinal microbiota, the population of microorganisms that inhabit the intestine, plays an important role in the intestinal morphology, immunity, nutrient digestion and absorption, and host health ${ }^{1-3}$. Many studies have demonstrated that intestinal microbiota participates in many metabolic pathways, such as lipid metabolism and amino acid synthesis ${ }^{4,5}$. The mechanism by which PEO promote growth of may be alter gut microflora and hence improved absorption of nutrients ${ }^{6}$, increase absorption of micronutrients in the small intestine ${ }^{7}$ and reducing the deleterious effects of the microbial metabolites ${ }^{8-10}$. There are many alternatives to antibiotics, such as acidifiers, probiotics, oligosaccharides, and plant extracts, which play a growth promoting role by regulating gut microbes in pig and poultry ${ }^{11}$.

Plant essential oils (PEO), which can be extracted from plants by steam distillation, extrusion, or solvent extraction ${ }^{12,13}$, serve as alternatives for antibiotics used in feed for their safety and limited residual effects ${ }^{5,13-15}$. Many studies have shown that PEO can decrease the number of E. coli and increase the amount of Lactobacilli in the intestine of broilers ${ }^{5,16-18}$. This has beneficial effects on the intestinal morphology and barrier, and the antioxidant in animals ${ }^{19}$. Also, Altop et al. (2018) reported the interactions of host gut-microbiota and co-metabolism 
on chickens that were fed with essential oils ${ }^{20}$. However, only a few studies have investigated the effects of PEO or antibiotics on the cecum microbiota and serum metabolites profile of the chicken.

In this study, the cecal microbial composition and metabolites in the cecum and serum of chickens that were fed with virginiaymicin (VIRG) or PEO were investigated through 16S rRNA gene sequencing and untargeted mass spectrometry (UPLC-Q-TOF/MS) metabolomic analysis. Comparing the differences in the cecal microbial composition and the potential chemical metabolites might help us understand how the dietary antibiotics or PEO alter the intestinal metabolism and health of the broiler chickens.

\section{Results}

Diversity and structure of the cecal microbiota. High-throughput sequencing obtained 176,636 quality-controlled reads from 18 cecum samples from three treatments (PEO group,VIRG group and CT group). After denoising, removing chimeras, and filtering low quality sequences, the sample had an average of 44,120 sequences, the average length of each sequence being 443 nucleotides. Based on the $97 \%$ identity level, these sequences were decomposed into 1,971 operational taxonomic units (OTUs). The 16S rRNA gene amplicon sequencing results were deposited in the Sequence Read Archive of the NCBI (accession number PRJNA553851).

The diversity of the cecal microbiota in the three groups are shown in Fig. 1. Compared to the CT and the PEO group, the Chaol, ACE, and Shannon diversity indices were observed to be significantly lower in the VIRG group. No differences in the diversity indices (Shannon, Chaol, and Observed species) were observed between the PEO and the CT group (Fig. 1A and Supplementary Table 1A). Principal Component Analysis based on EUCLIDEAN distance showed that the cecal microbes, among the three treatments of CT, PEO, and VIRG groups, formed distinct clusters. The treatment groups were well separated with $48.96 \%$ and $27.11 \%$ variation by the principal components PC1 and PC2, respectively (Fig. 1B). PLS-DA model shows significant separation and discrimination, indicating that the PLS-DA model can be used to identify differences between groups.

Dietary treatment with PEO or VIRG (Fig. 2 and Supplementary Table 1B) resulted in a change in the cecal microbial composition. At the phylum level (Fig. 2A), Firmicutes was the most predominant phylum (more than 60\%), followed by Bacteroidetes (10-20\%), Actinobacteria (2-6\%), and Verrucomicrobia (1\%). Additionally, there were more than $6 \%$ unclassified bacteria in the cecal microbiota of chickens. Compared to the CT group, the relative abundance of phyla Bacteroidetes increased while that of phyla Firmicutes decreased in the cecal microbiota of the PEO and the VIRG groups. At the genus level (Fig. 2B), Lactobacillus, Faecalibacterium, Bacteroidaceae, and unclassified Rikenellaceae were the predominant genus in the cecal microbiota of the chicken. The relative abundance of Lactobacillus was lower, and that of unclassified Rikenellaceae was higher in the cecal microtia of the PEO group. The genus Bacteroides was higher in the VIRG group than in the CT group.

The LEfSE test results (Fig. $2, \mathrm{LDA}>2, \mathrm{P}<0.05$ ) showed that the proportion of genera Alistipes, unclassified Rikenellaceae, Anaeroplasma, unclassified Bacillaceae, and Roseburia were higher in the cecal microbiota of the PE group (Fig. 2C). The relative abundance of phyla Bacteroidetes and the genus of Bacteroides, unclassified Enterobacteriaceae, unclassified Lachnospiraceae, and Anaerofustis were higher in cecal microbiota of VIRG group. The proportion of phyla Firmicutes and Actionbacteria and genus of Lactobacillus, streptococcus, unclassified Erysipelotrichaceae, coriobacteriaceae, and streptococcaceae were higher in cecal microbiota of CT group (Fig. 2C).

Effects of dietary plan essential oils or virginiamycin on caecum and serum metabolites. A non-targeted LC-MS-based metabolomics platform was used to analyze the cecum contents and the serum metabolite profiles of chicken fed supplemented with PEO or VIRG. According to the variable importance in the projection (VIP) value $>1$, in $95 \%$ jack-knifed confidence intervals and $\mathrm{P}<0.05$, detailed information about the different biomarker metabolites has been shown in Supplementary Table 2. Compared to the CT group, 102 different metabolites with 53 LC-MS/MS(ESI+) and 49 LC-MS/MS(ESI-), were detected in cecum contents of the PEO group; 81 different metabolites (30 ESI+ and 51 ESI-) were detected in the serum of the PEO group. VIRG group have 217 different metabolites (96 ESI+ and 121 ESI-) in cecum contents, and 142 different metabolites (82 ESI+ and 60 ESI-) in the serum as compared to the CT group. There were 305 different metabolites (148 ESI+ and 157 ESI-) in cecum contents, and 119 different metabolites (58 ESI+ and 61 ESI-) in the serum between PEO and VIRG groups.

Next, these different metabolites were conclusively identified using the in-house databases. Figure 3 shows the different metabolites in the cecal contents and serum. Compared to the CT group, dietary supplementation with PEO resulted in six more lipid metabolites and two more Carbohydrate metabolites in the cecal contents. These included 1-Oleoyl-sn-glycero-3-phosphocholine (LysoPC(18:1(9Z))), 1-Palmitoyl-2-hydroxy-sn-glycero3-phosphoethanolamine (LysoPE(16:0/0:0)), 1-Palmitoyl-sn-glycero-3-phosphocholine, Glycocholic acid, Caprylic acid, and Chenodeoxycholate. Amino acid related metabolites (Isoleucyl-valine, Hydroxyproline, beta-Homoproline) decreased in the cecal contents of chicken fed with PEO (Fig. 3A). Additionally, the PEO treatment resulted in 6 more lipid metabolites (Palmitic acid, 9,10-DiHOME, etc.) and six more Carbohydrate metabolites (such as D-Mannose) in the serum of chickens. Compared to the CT group,some metabolites (Citrate, taurine, nicotinamide, and urea) were lower in the serum of PEO group (Fig. 3C). Compared to the VIRG group, PEO group increased 11 lipid metabolites (Arachidonic Acid, docosapentaenoic acid, linoleic acid, 1-Palmitoylglycerol, dodecanoic acid(C12), myristic acid(C14), caprylic acid(C8), $\beta$-hydroxy butyric acid (C4), FC > 1.5) and Cofactors and Vitamins (Pyridoxal (VB6) and Pantothenate) in the cecal contents (Fig. 3E).

Compared to the CT group, dietary supplement with virginiamycin increased the levels of (S)-Equol, LysoPE (16:0/0:0), Caproic acid, D-Maltose, and ribitol in the cecum of the chicken. However, 9 other metabolites including nucleotides (Cytosine, Thymine, Ademine, and Uracil), Myristoleic acid, and Pyridoxal (Vitamin B6) decreased (Fig. 3B). About 10 lipid metabolites such as oleic acid, 9,10-DiHOME, 1-Stearoyl2-oleoyl-sn-glycerol-3-phosphocholine (SOPC), Adynerin, betaine, DL-lactate, and dopamine were higher in the 
A

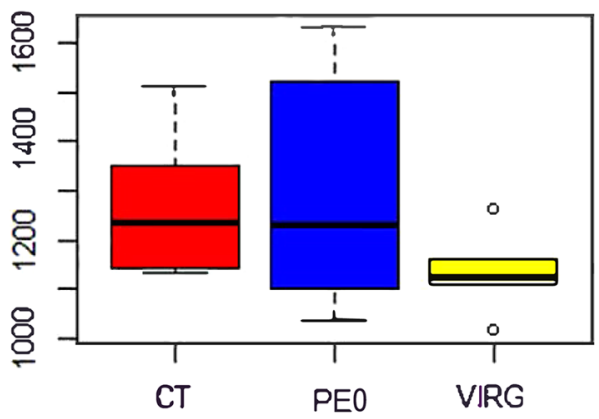

Shannon

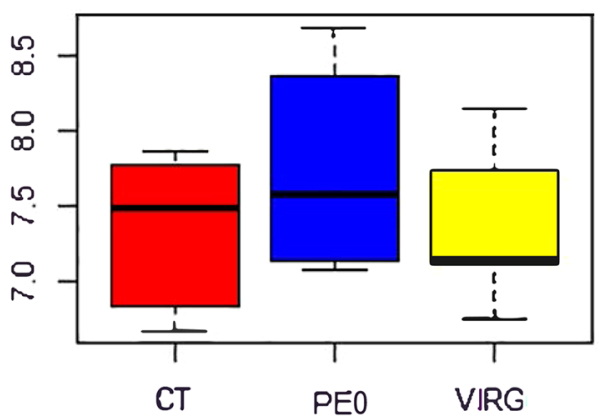

B

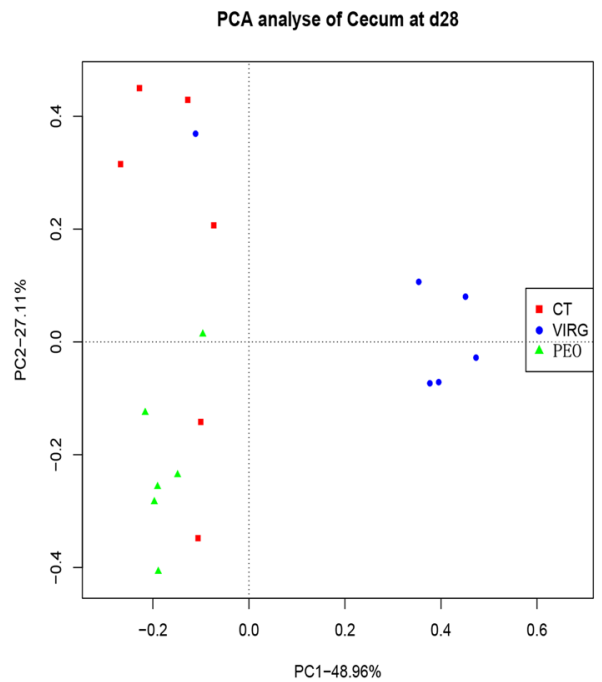

ACE

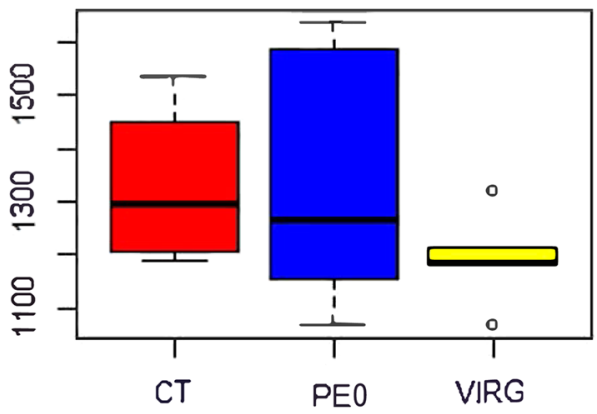

OTU

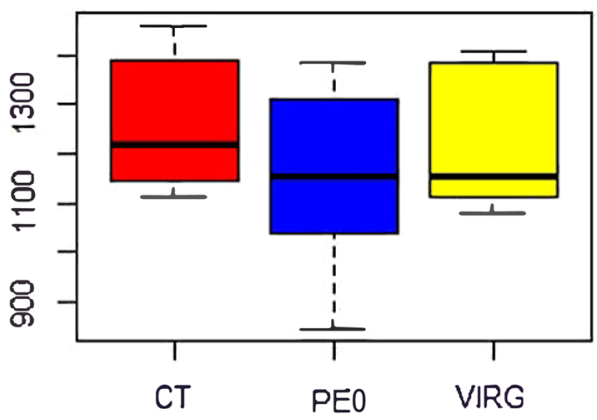

PLS-DA of Caecum at d28

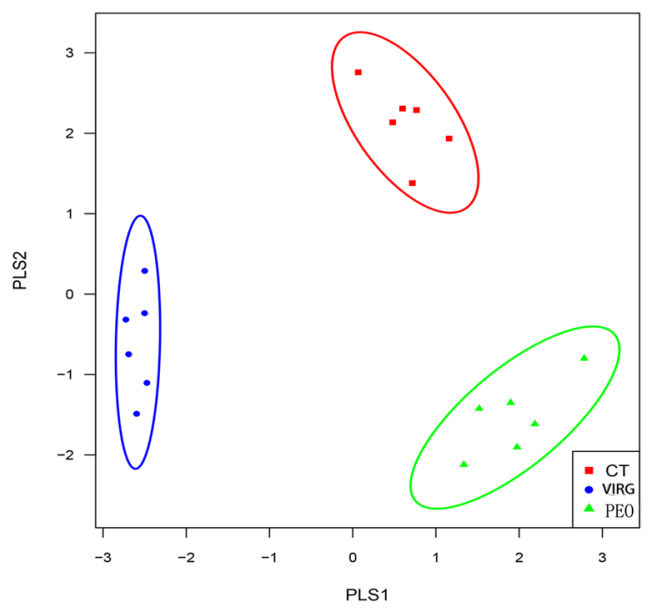

Figure 1. Differences in bacterial community diversity, richness, and structures in the cecum of broiler chickens fed without or with PEO or VIRG. (A) Community diversity and richness among CT, PEO, and VIRG group. (B) Principal components analysis (PCA) of the bacterial community structure among CT, PEO, and VIRG group. Each symbol represents each gut microbiota. Red symbols represented CT group, blue symbols represented PEO group, and brown symbols represented VIRG group. CT: the basal diet; PEO: the basal diet supplemented with plant extracts; VIRG: the basal diet supplemented with virginiamycin. PLS-DA score plots showed significantly separated clusters between CT, PEO, and VIRG group. 
A

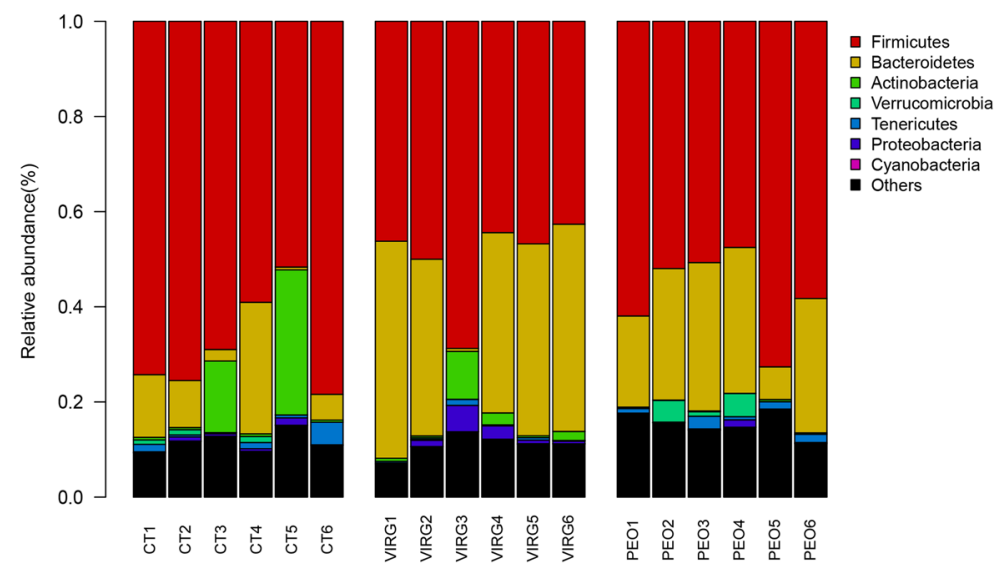

B

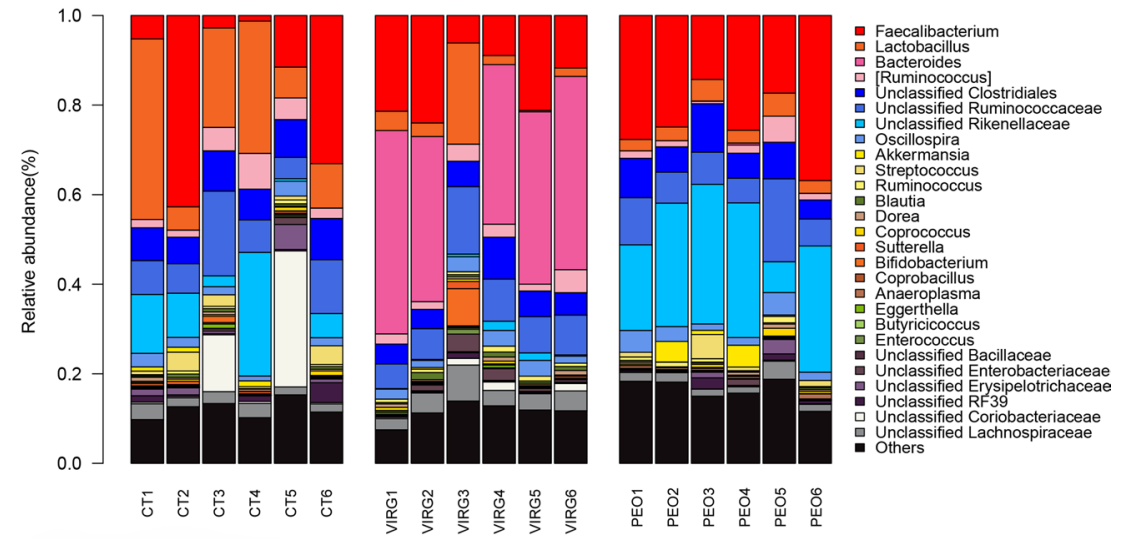

C

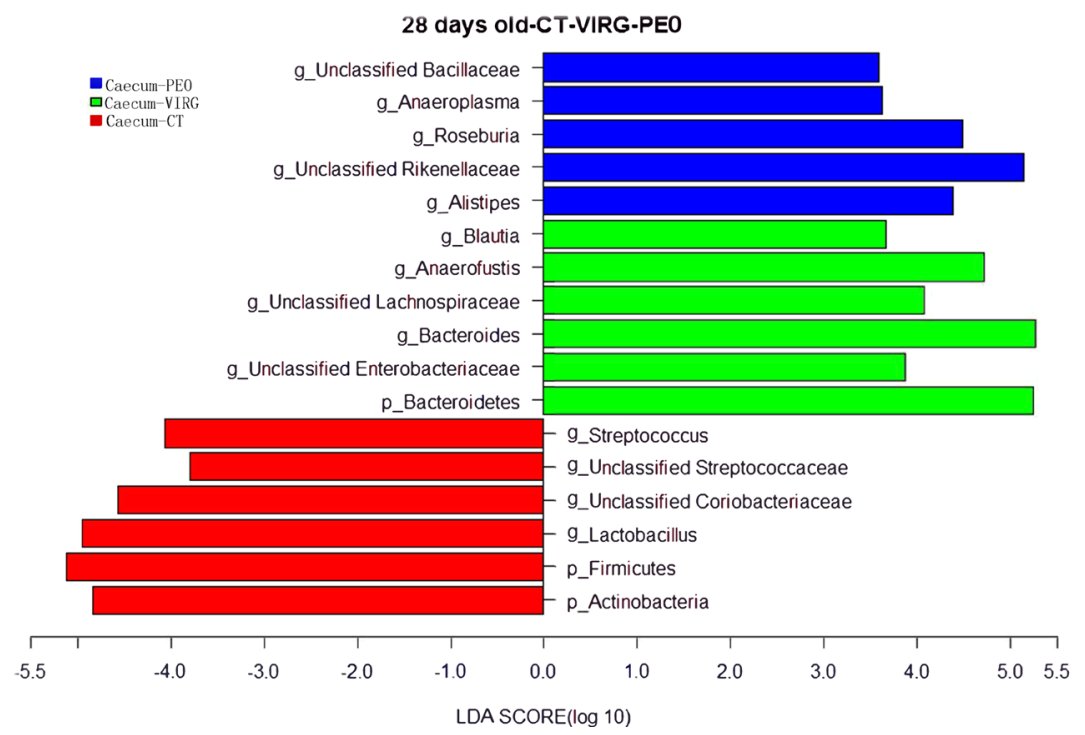

Figure 2. Changes of microbial composition in the cecum of broiler chickens fed without or with PEO or VIRG. Microbial composition at the phylum level (A) and genus level (B) each bar represents the relative abundance of each bacterial taxa of chicken. Bacterial taxa significantly differentiated between CT, PEO group, and VIRG group $(\mathbf{C})$ and was identified by linear discriminant analysis coupled with effect size (LEfSe) using the default parameters. CT: the basal diet; PEO: the basal diet supplemented with plant extracts; VIRG: the basal diet supplemented with virginiamycin.

serum of VIRG group. However, four metabolites (1-Palmitoyl-sn-glycero-3-phosphocholine,cytidine, taurine, cysteine-s-sulfate) were lower in the serum of chicken fed with virginiamycin (Fig. 3D). Only 10 metabolites were different in serum of chickens between PEO and VIRG groups (Fig. 3F). 
A

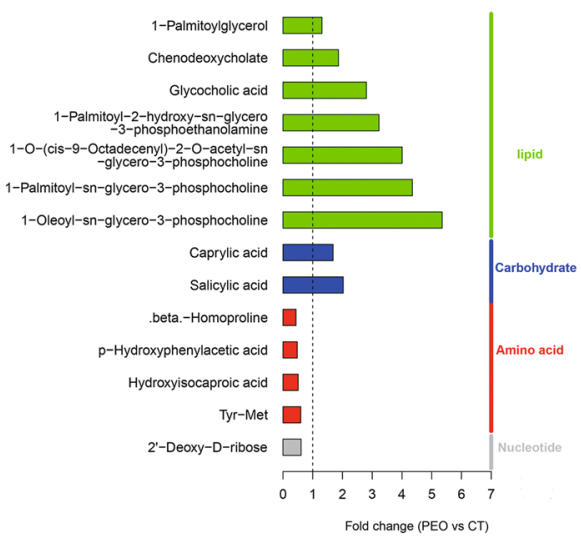

C

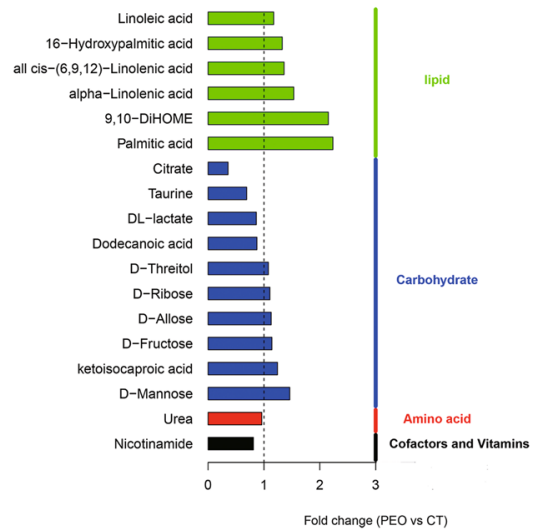

$\mathrm{E}$

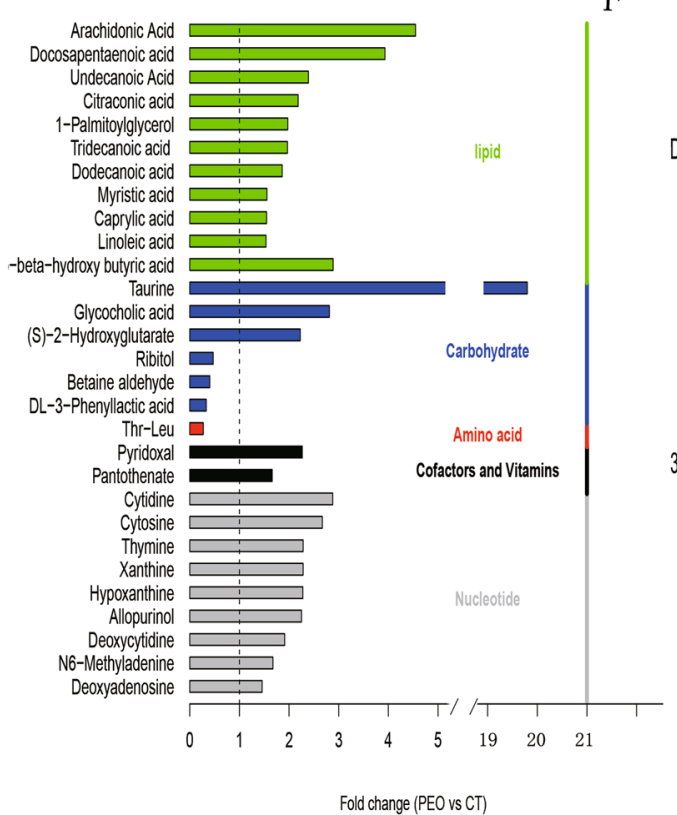

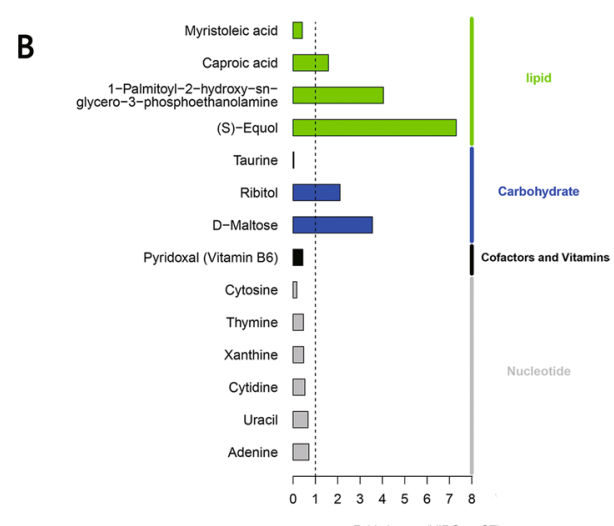

Fold change (VIRG vs CT)

D

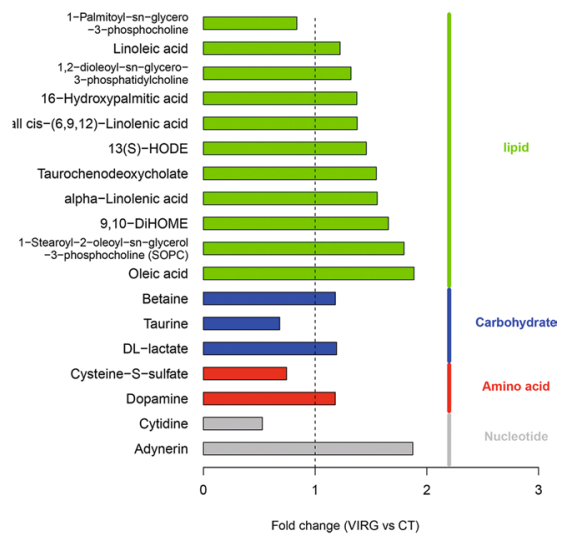

$\mathrm{F}$

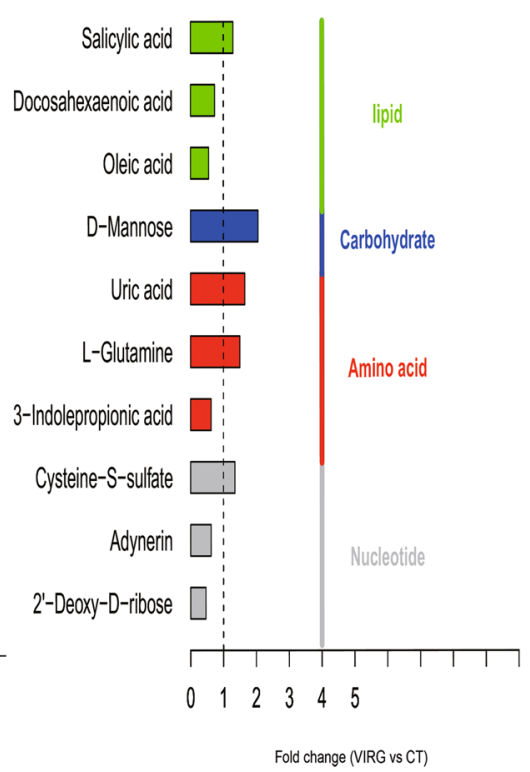

Figure 3. Significantly differential metabolites in the cecum and serum of broiler chickens fed without or with PEO or VIRG. Differential metabolites on PEO vs. CT in the cecum (A), VIRG vs. CT and VIRG in the cecum (B); differential metabolites on PEO vs. CT in the serum (C), VIRG vs. CT and VIRG in the serum (D). Metabolites accountable for class discrimination with VIP $>1$ and $\mathrm{P}<0.05$ were listed. CT: the basal diet; PEO: the basal diet supplemented with plant extracts; VIRG: the basal diet supplemented with virginiamycin.

The relationship of different relative abundance of bacteria in the cecal microbiota with cecal and serum metabolic. Pearson's correlation analyses showed that the relative abundance of different bacteria (LEfSE) at the genus and phylum level in the cecal microbiota were found to be closely associated to the concentration of specific microbial metabolites in the cecum and serum of chickens (Fig. 4). The high proportion of bacteria in the cecal microbiota of the PEO group was positively correlated with 1-Palmitoylglycerol, myristoleic 
A

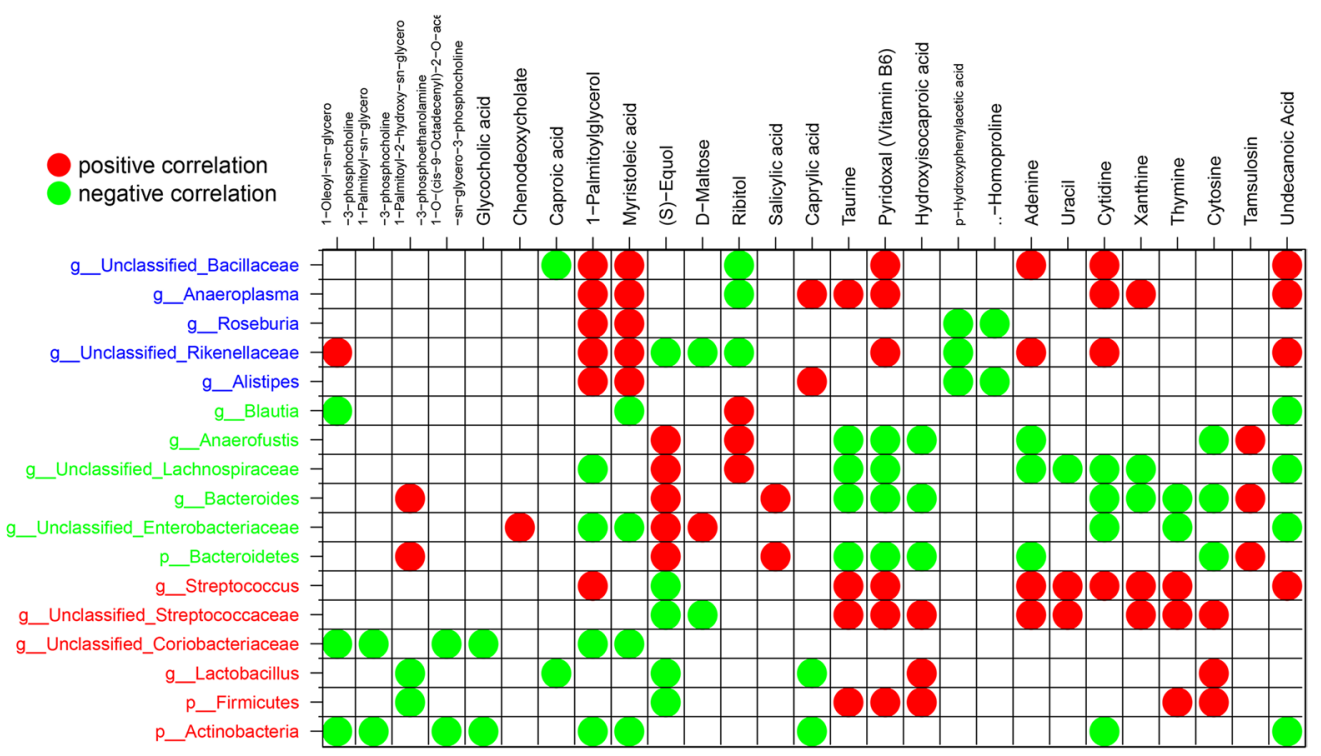

B

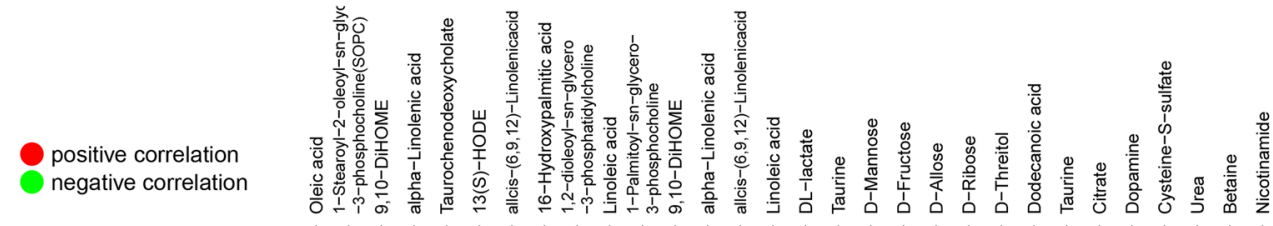

HPE

g_Unclassified_Bacillaceae

g_Anaeroplasma g_Roseburia g_Unclassified_Rikenellaceae g_Alistipes

g_Blautia g_Anaerofustis

CT

g_Unclassified_Coriobacteriaceae

g_Lactobacillus

P_Actinobacteria

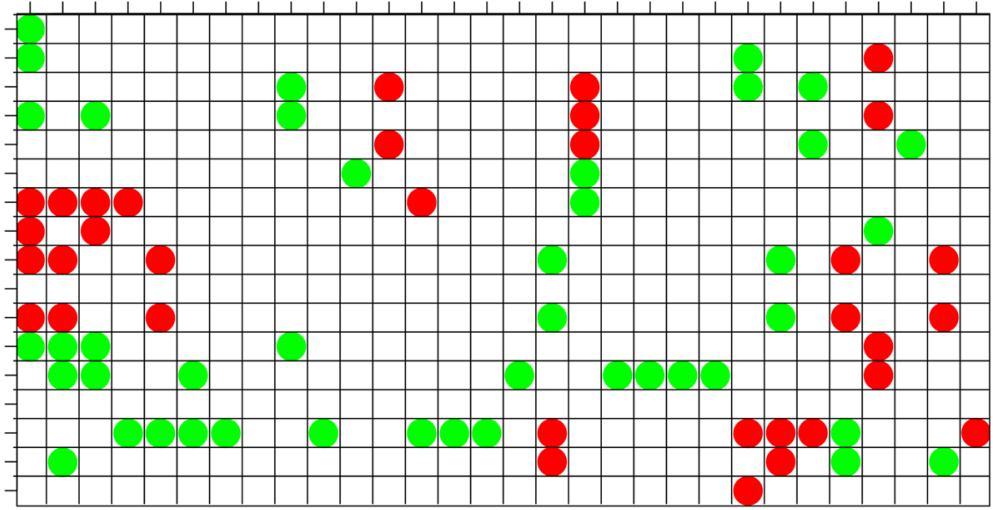

Figure 4. Correlation between microbiota and metabolites in the cecum $(\mathbf{A})$ and serum $(\mathbf{B})$ of broiler chickens fed without or with dietary PEO or VIRG. The color was according to the Spearman correlation coefficient distribution. Red represented significant positive correlation $(\mathrm{P}<0.05)$, blue represented significantly negative correlation $(\mathrm{P}<0.05)$, and white represented that the correlation was not significant $(\mathrm{P}>0.05)$. CT: the basal diet; PEO: the basal diet supplemented with plant extracts; VIRG: the basal diet supplemented with virginiamycin.

acid, and Pyridoxal (Vitamin B6) in the cecum and D-Mannose in the serum. Additionally, the relative abundance of unclassified Rikenellaceae positively correlated with 1-Oleoyl-sn-glycero-3-phosphocholine, adenine, cytidine, and undecanoic acid in cecum (Fig. 4A) and negatively correlated with (S)-Equol, p-Hydroxyphenylacetic acid, and ribitol in the cecum and in the serum of chicken (Fig. 4B). The genus Alistipes positively correlated with 1-Palmitoylglycerol, myristoleic acid, and caprylic acid in the cecum and D-Mannose and 9,10-DiHOME in the serum of chicken, negatively correlated with $\beta$-Homoproline and p-Hydroxyphenylacetic acid in caecum and in citrate and urea in serum.

The high proportion of bacteria in the cecal microbiota of the VIRG group positively correlated with ribitol and (S)-Equol in the cecum and oleic acid, SOPC, and adynerin in the serum, and negatively correlated with cytidine, hydroxyisocaproic acid, and Pyridoxal (Vitamin B6) in the cecum. Additionally, the relative abundance of phylum Bacteroidetes and genera Bacteroides positively correlated with LysoPE(16:0/0:0), salicylic acid, and tamsulosin in the cecum and betaine, taurochenodeoxycholate, and dopamine in the serum while negatively correlating with hydroxyisocaproic acid, cytosine, taurine, and Pyridoxal (Vitamin B6) in the cecum and taurine in the serum. The relative abundance of phylum Firmicutes positively correlated with hydroxyisocaproic acid, cytosine, taurine, and Pyridoxal (Vitamin B6) in the cecum and taurine in the serum, while negatively 


\begin{tabular}{|c|c|c|c|c|}
\hline & Metabolites(cpdName) & Map.Name & Map_ID & Pathway hierarchy \\
\hline \multirow{3}{*}{ caecum } & $\begin{array}{l}\text { Chenodeoxycholate }\left(1.86 \mathrm{FC}^{1}\right) \text {, Glycocholic acid } \\
(2.80 \mathrm{FC})\end{array}$ & Primary bile acid biosynthesis & map00120 & Lipid metabolism \\
\hline & $\begin{array}{l}\text { p-Hydroxyphenylacetic acid( } 0.48 \mathrm{FC}) \text {, Salicylic } \\
\text { acid(2.02FC) }\end{array}$ & Phenylalanine metabolism & map00360 & Amino acid metabolism \\
\hline & Thymine(0.63FC), Deoxycytidine (1.62FC) & Pyrimidine metabolism & map00240 & Nucleotide metabolism \\
\hline \multirow{6}{*}{ serum } & D-Mannose(1.46FC), D-Allose(1.13FC) & $\begin{array}{l}\text { Fructose and mannose } \\
\text { metabolism }\end{array}$ & map00051 & Carbohydrate metabolism \\
\hline & $\begin{array}{l}\text { Linoleic acid(1.44FC), alpha-Linolenic } \\
\text { acid(1.53FC), all cis- } 6,9,12) \text {-Linolenic } \\
\text { acid(1.36FC), Palmitic acid(2.23FC) }\end{array}$ & $\begin{array}{l}\text { Biosynthesis of unsaturated } \\
\text { fatty acids }\end{array}$ & map01040 & \multirow{3}{*}{ Lipid metabolism } \\
\hline & $\begin{array}{l}\text { Linoleic acid(1.46FC), all cis- }(6,9,12) \text {-Linolenic } \\
\operatorname{acid}(2.23 \mathrm{FC})\end{array}$ & Linoleic acid metabolism & map00591 & \\
\hline & Dodecanoic $\operatorname{acid}(0.87 \mathrm{FC})$, Palmitic acid(1.36FC) & Fatty acid biosynthesis & map00061 & \\
\hline & $\begin{array}{l}\text { Taurine(0.69FC), D-Mannose(1.46FC), } \\
\text { D-Allose(1.13FC), D-Ribose(1.105FC), } \\
\text { Urea(0.96FC), } \\
\text { Betaine(1.43FC) }\end{array}$ & ABC transporters & map02010 & Membrane transport \\
\hline & D-Mannose(1.46FC) & Lysosome & map04142 & Transport and catabolism \\
\hline
\end{tabular}

Table 1. The relevant KEGG pathways enriched by metabolites between $\mathrm{PEO}$ with $\mathrm{CT}$ group. ${ }^{1} \mathrm{FC}=$ Fold change.

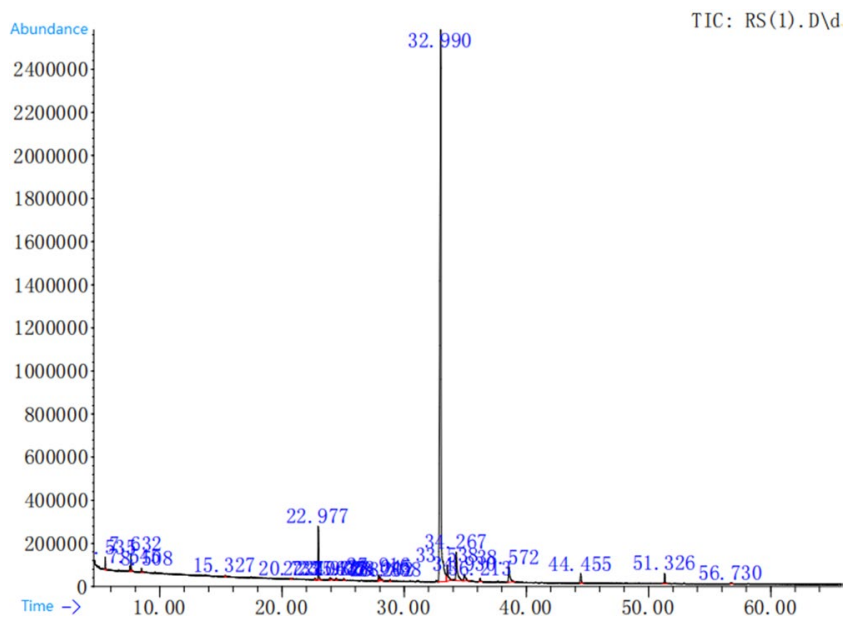

Figure 5. Gas chromatography-mass spectrometry total ion chromatogram of PEO. Peaks are representative of the mass of the compounds present in PEO: Cinnamical dehyde $(\mathrm{RT}=33.6$, Area $=78.3 \%)$, Isophorone $(\mathrm{RT}=22.9$, Area $=4.23 \%)$.

correlating with LysoPE(16:0/0:0) and (S)-Equol in the cecum and SOPC, betaine, and dopamine in the serum. The relative abundance of genera Lactobacillus positively correlated with hydroxyisocaproic acid and cytosine in the cecum and taurine, dodecanoic acid, citrate, and nicotinamide in the serum, while negatively correlating with LysoPE(16:0/0:0), (S)-Equol, and caprylic acid in the cecum and alpha-linolenic acid, cis-(6,9,12)-linolenic acid, linoleic acid, taurochenodeoxycholate, 13(S)-HODE, and dopamine in the serum.

Effects of dietary plan essential oils or virginiamycin on KEGG pathway in caecum and serum. Based on these metabolites (VIP $>0.1, \mathrm{P}<0.05$ ), the software Metaboanalyst 3.0 was used to enrich the relevant KEGG pathways. The relevant KEGG pathways enriched by metabolites between PEO within the CT group are shown in Table 1. As compared to the CT group, three relevant KEGG pathways were significantly enriched for these metabolites in the cecum of the PEO group, including cecal primary bile acid biosynthesis (up-regulated by Chenodeoxycholate (1.86 FC (Fold change)) and Glycocholic acid (2.80 FC)); cecal Phenylalanine metabolism (up-regulated by Salicylic acid (2.02 FC) and Pyrimidine metabolism. There were six relevant KEGG pathways enriched in the serum of chickens of the PEO group (Fig. 5C), including fructose and mannose metabolism (up-regulated by D-mannose (1.46 FC) and D-allose (1.13 FC)), biosynthesis of unsaturated fatty acids, Linoleic acid metabolism, and serum fatty acid biosynthesis were enriched.

The relevant KEGG pathways enriched by metabolites between VIRG within the CT group are shown in Table 2. Dietary supplement virginiamycin altered 10 cecal KEGG pathways and seven serum KEGG pathways of chicken. D-mannose and L-leucine up-regulated the KEGG pathway of Lysosome and the mTOR signaling pathway respectively. D-glucosamine 6-phosphate up-regulated and L-alanine down-regulated the KEGG pathway of 


\begin{tabular}{|c|c|c|c|c|}
\hline & Metabolites(cpdName) & Map.Name & Map_ID & Pathway hierarchy \\
\hline \multirow{10}{*}{ caecum } & Thymine $\left(0.46 \mathrm{FC}^{\mathrm{l}}\right)$, Cytidine(0.53FC), Uracil(0.69FC), Pseudouridine Cytosine(0.17FC), & Pyrimidine metabolism & map00240 & Nucleotide metabolism \\
\hline & D-Maltose(3.55FC), Taurine(0.73FC), L-Leucine L-Alanine(0.54FC), D-Mannose(6.91FC) & ABC transporters & map02010 & Membrane transport \\
\hline & Dodecanoic acid(0.57FC), Myristic acid(0.41FC), Oleic acid (0.46FC) & Fatty acid biosynthesis & map00061 & Lipid metabolism \\
\hline & L-Alanine(0.54FC) D-glucosamine 6-phosphate(1.63FC) & $\begin{array}{l}\text { Alanine, aspartate and } \\
\text { glutamate metabolism }\end{array}$ & map00250 & Amino acid metabolism \\
\hline & Taurine(0.73FC), L-Alanine(0.54FC) & $\begin{array}{l}\text { Taurine and hypotaurine } \\
\text { metabolism }\end{array}$ & map00430 & \multirow{2}{*}{$\begin{array}{l}\text { Metabolism of other amino } \\
\text { acids }\end{array}$} \\
\hline & Uracil(0.56FC), Pantothenate(0.63FC) & beta-Alanine metabolism & map00410 & \\
\hline & 4-Pyridoxic acid(1.68FC), Pyridoxal(0.43FC) & Vitamin B6 metabolism & map00750 & \multirow{2}{*}{$\begin{array}{l}\text { Metabolism of cofactors } \\
\text { and vitamins }\end{array}$} \\
\hline & Uracil(0.56FC), Pantothenate(0.63FC) & $\begin{array}{l}\text { Pantothenate and CoA } \\
\text { biosynthesis }\end{array}$ & map00770 & \\
\hline & D-Mannose(6.91FC) & Lysosome & map04142 & Transport and catabolism \\
\hline & L-Leucine(2.34FC) & mTOR signaling pathway & map04150 & Signal transduction \\
\hline \multirow{7}{*}{ serum } & Citrate(0.68FC), L-Malic acid(0.83FC) & Citrate cycle (TCA cycle) & map00020 & Carbohydrate metabolism \\
\hline & $\begin{array}{l}\text { Oleic acid(1.88FC), alpha-Linolenic acid(1.55FC), Linoleic acid(1.41FC), all cis- }(6,9,12) \text { - } \\
\text { Linolenic acid(1.37FC) }\end{array}$ & $\begin{array}{l}\text { Biosynthesis of unsaturated } \\
\text { fatty acids }\end{array}$ & map01040 & \multirow{4}{*}{ Lipid metabolism } \\
\hline & $\begin{array}{l}\text { Linoleic acid(1.41FC), all cis-(6, 9, 12)-Linolenic acid(1.37FC), 1-Stearoyl-2-oleoyl-sn-glycerol } \\
\text { 3-phosphocholine (SOPC)(1.79FC), }\end{array}$ & Linoleic acid metabolism & map00591 & \\
\hline & $\begin{array}{l}\text { alpha-Linolenic acid(1.55FC), 1-Stearoyl-2-oleoyl- } \\
\text { sn-glycerol 3-phosphocholine (SOPC)(1.79FC) }\end{array}$ & $\begin{array}{l}\text { alpha-Linolenic acid } \\
\text { metabolism }\end{array}$ & map00592 & \\
\hline & Taurine(0.68FC), Taurochenodeoxycholate(1.547FC), & $\begin{array}{l}\text { Primary bile acid } \\
\text { biosynthesis }\end{array}$ & map00120 & \\
\hline & Urea(0.62FC), Cytidine(0.53FC), Methylmalonic acid(0.52FC), & Pyrimidine metabolism & map00240 & Nucleotide metabolism \\
\hline & L-Tyrosine(1.17FC) & Melanogenesis & map04916 & Endocrine system \\
\hline
\end{tabular}

Table 2. The relevant KEGG pathways enriched by metabolites between VIRG with CT group. ${ }^{1} \mathrm{FC}=$ Fold change.

alanine, aspartate, and glutamate metabolism. Many metabolites such as dodecanoic acid, myristic acid, oleic acid, taurine, L-alanine, uracil, pantothenate, and Pyridoxal down-regulated the cecal KEGG pathway of fatty acid biosynthesis, beta-alanine metabolism, taurine and hypotaurine metabolism, pantothenate, and CoA biosynthesis. In serum, lipid metabolism KEGG pathway were enriched in the serum of the chicken feed with VIRG, including biosynthesis of unsaturated fatty acids (up-regulated by oleic acid (1.88 FC), alpha-linolenic acid (1.55 FC), linoleic acid (1.41 FC), all cis- $(6,9,12)$-linolenic acid (1.37 FC), linoleic acid metabolism (linoleic acid (1.41 FC), all cis- $(6,9,12)$-linolenic acid (1.37 FC), SOPC (1.79 FC), and alpha-linolenic acid metabolism (alpha-linolenic acid (1.55 FC), SOPC (1.79 FC)). Primary bile acid biosynthesis was increased by Taurochenodeoxycholate (1.547 FC) and decreased by Taurine (0.68 FC). Then, citrate cycle (Citrate (0.68 FC), L-Malic acid (0.83 FC)), and Pyrimidine metabolism (Urea (0.62 FC), Cytidine (0.53 FC), Methylmalonic acid (0.52 FC), in serum were decreased.

\section{Discussion}

Since the ban on antibiotic growth-promotion (AGP) in many countries, several effective alternatives to AGPs have been developed in recent years. One of them, PEO or phytogenic additives are considered as appropriate candidates due to their safety benefits ${ }^{15,21}$. Our previous study has shown that dietary supplementation with PEO improved the growth and feed efficiency in broiler chickens ${ }^{22}$, which was consistent with other studies (reviewed by Windisch et al., 2008; Zeng et al., 2015; and Diaz-Sanchez et al., 2015) ${ }^{14,23,24}$. PEO might promote growth by altering gut microflora and hence improving absorption of nutrients ${ }^{6}$, increasing the absorption of micronutrients in the small intestine ${ }^{7}$, stimulating the production and activity of digestive enzymes (such as trypsin and amylase ${ }^{25,26}$, and stimulating digestive and physiology metabolism ${ }^{27}$.

This study shows that both PEO and VIRG treatments increased the relative abundance of phyla Bacteroidetes and decreased the relative abundance of phyla Firmicutes and genera of Lactobacillus and streptococcus in the cecal microbiota of chickens. Meanwhile, the relative abundance of phyla Bacteroidetes and genera Alistipes, Roseburia, and unclassified Rikenellaceae increased in the cecal microbiota of the PEO group. These finding were consistent with previous studies that reported that PEO decreased the number of Lactobacilli ${ }^{28,29}$. Next, some researchers reported that there was a significant increase in the Lactobacillus in the intestines of chickens fed with PEO or Macleaya cordata extracts ${ }^{18}$. These differences may be due to the difference in the source and breed of PEOs, feeding patterns, and sampling parts of the chicken ${ }^{14}$.

The intestinal microbiota plays multiple roles in the intestinal morphology, immunity, nutrient absorption and metabolism, and host health ${ }^{1,2}$. Phyla Bacteroidetes are gram-negative bacteria that ferment polysaccharides and other indigestible carbohydrates and produce short-chain fatty acids (SCFAs) that are gut-friendly. Bacteroidetes are related to fat accumulation in chickens ${ }^{30}$. Alistipes and unclassified Rikenellaceae, which belong to phylum Bacteroidales, are generally considered beneficial to the host gut ${ }^{31}$. Alistipes can produce succinic acid and other long-chain fatty acids such as $\mathrm{C}_{1} 5^{32}$. Rikenellaceae can produce propionic and succinic acids by fermentation of glucose, lactose, mannose, and melibiose, and formed the iso-methyl branched-chain fatty acid or long chain saturated acids ${ }^{33}$. Genus Roseburia belongs to the Lachnospiraceae family, a butyrate-producing organism, with a high capacity to form conjugated linoleic acid from linoleic acid ${ }^{34}$. Our Pearson's correlation analyses revealed 
that phylum Bacteroidetes were positively correlated with LysoPE (16:0/0:0) and (s)-equol in the cecum and betaine, taurochenodeoxycholate, and dopamine in the serum, while negatively correlating with hydroxyisocaproic acid, cytosine, and taurine in the cecum and taurine in the serum. Moreover, unclassified Rikenellaceae and Alistipes were positively correlated with 1-Palmitoylglycerol, myristoleic acid, and Pyridoxal (Vitamin B6) in the cecum and D-Mannose in the serum. These metabolites participated in many biological functions. e.g. LysoPE (16:0/0:0), a lysophospholipid, that serves important signaling functions. Equol may enhance the actions of soy isoflavones, due to its greater affinity for estrogen receptors and have unique antiandrogenic and antioxidant activities ${ }^{5}$. Dopamine is a major transmitter in the extrapyramidal system of the brain and is important in regulating movement. A family of receptors (dopamine receptors) mediates action, which plays a major role in the reward-motivated behaviour ${ }^{35}$. The enrichment of the relative abundance of Phyla Bacteroidetes and genera Alistipes, unclassified Rikenellaceae might be related with biosynthesis of fatty acid and lipid metabolism. Recently, some researchers have reported that the increase in the proportion of Phyla Bacteroidetes is related to promoting animal growth performance. On the contrary, Although Lactobacillus is considered to be a beneficial probiotic to the host intestinal health and growth ${ }^{36}$, our results showed that the proportion of Lactobacillus in the cecum negatively correlated with lipid metabolites, such as LysoPE (16:0/0:0) and (S)-Equol in the cecum and alpha-linolenic acid, all cis- $(6,9,12)$-linolenic acid, linoleic acid, taurochenodeoxycholate, and dopamine in the serum; and positively correlated with some metabolites which accelerated lipid peroxidation (such as hydroxyisocaproic acid and cytosine), down-regulated KEGG pathway related to primary bile acid biosynthesis and citrate cycle (TCA cycle). These results were consistent with De Boever et al. ${ }^{37}$, who reported that Lactobacillus impaired lipid absorption and consequently resulted in the dietary energy losses ${ }^{37}$. Recently, some reports showed that some Lactobacillus strains had a negative influence on the growth performance ${ }^{30,38}$, some strains of Lactobacillus are retailed as weight loss probiotics while others are reported with the ability to reduce obesity for increasing appetite and feed consumption ${ }^{39,40}$. Therefore, alteration of intestinal flora may result to improve of animal performance.

Metabolomic analysis revealed that chenodeoxycholate and glycocholic acid up-regulated the KEGG pathway of primary bile acid biosynthesis in the cecal contents of PEO group, Primary bile acids are also critical to the digestion and absorption of $\mathrm{fat}^{41}$. In the blood of chickens of PEO group, six lipid metabolites (such as LysoPC (18:1(9Z)) and LysoPE (16:0/0:0)) enriched fatty acid biosynthesus, Lyso PCs perform essential functions in the lipid metabolism of organisms ${ }^{9,42}$; 1-Palmitoylglycerol is the biosynthetic precursor of phosphatidic acid: the major component of lysophosphatidic acid, which is a pluripotent lipid mediator which controls growth, motility, and differentiation ${ }^{43}$. In addition, D-Mannose up-regulated KEGG pathway of fructose and mannose metabolism and $\mathrm{ABC}$ transporters in serum of the PEO group. ABC transporters utilize the energy of ATP binding and hydrolysis to transport various substrates across cellular membranes ${ }^{44}$. Above all, dietary supplementation with PEO alters the cecal and blood metabolic pathways, The biosynthesis pathways of fatty acids and unsaturated fatty acids that are closely related to host lipid metabolism might be an important mechanism for growth promoters of $\mathrm{PEO}^{13}$.

The mechanism of improving performance of AGPs is related to reduced incidences of subclinical infections, stability of the microbial ecology, thinning of the intestinal wall, suppression of inflammation, and the reduction of bioamines and toxins produced by the bacteria ${ }^{45,46}$. Recently, extensive research on the effects of antibiotics on intestinal biochemicals has been carried out using culture-independent methods and metabolomic analysis ${ }^{9,10,47}$. Many reports showed that the relative abundance of phyla Bacteroidetes increased and that of genus Lactobacillus decreased in the intestines of chickens after the administration of antibiotic ${ }^{9,10,47-50}$, Higher numbers of lactobacilli were previously implicated in broiler growth depression due to competition in nutrient uptake or impaired fat absorption ${ }^{30,48}$. Some other studies showed that proportion of phyla Firmicutes and genus of Lactobacillus increased in the gut of the chicken after administration of antibiotics ${ }^{51-53}$. Metabolomic analysis showed that 4-EOTC treatment improved the synthesis of lysophosphatidylcholine (LysoPC), as indicated by the lipid biomarkers LysoPC (16:0), LysoPC (18:3), LysoPC (20:3), and LysoPC (20:4) in the blood of Wistar rats. VIRG treatment altered 218 biochemicals (156 increased, 62 decreased) in the ileum of chickens, including many long chain saturated and polyunsaturated fatty acids; several lysophospholipids also increased in the process ${ }^{10}$. This observation was corroborated by our data that VIRG treatment increased the relative content of phyla Bacteroidetes and decreased that of genus Lactobacillus in the cecum, enriched lipid metabolites and biosynthesis of fatty acid and linoleic acid, and reduced some metabolites (such as urea) that may be toxic or harmful to growth of host.

Compared to the VIRG group, PEO group also increased the content of D-Mannose and arachidonic acid, docosapentaenoic acid, linoleic acid in the cecum of chickens. These metabolites are essential fatty acids with several biological functions: as the precursor that are metabolized by various enzymes to a wide range of bioactive components for growth and proper health ${ }^{54}$. Arachidonic acid is catalyzed by cyclooxygenase (COX) to generate prostaglandins (PGs), which regulate fever, inflammation, smooth muscle contraction, and translocation of water and salt in the kidney ${ }^{55,56}$. In addition, PEO treatment increases the biosystem of Pyridoxal (VB6) and Pantothenate in the cecal contents. Pyridoxal participates protein and sugar metabolism ${ }^{57}$, and the active form of VB6 is linked with adipogenesis ${ }^{58}$. These results indicate that PEO might alter different lipid metabolites and vitamins from AGP.

In summary, this study investigated the microbial profile and metabolites in the cecum and serum of chickens fed with PEO or VIRG by combined microbiome and metabolomic analysis. Our results demonstrated that PEO treatment increased the relative abundance of phyla Bacteroidetes and genera Alistipes, unclassified Rikenellaceae and decreased that of phyla Firmicutes and genus of Lactobacillus in the cecum. Many Lipid metabolites and KEGG pathway of fatty acid biosynthesis were enriched in cecum and serum of chickens in the PEO and VIRG group. These finding provide better understanding of the mechanism of promoting performance of PEO or VIRG, which could further provide useful information for developing an effective and safe alternative to AGP in poultry industry. 


\begin{tabular}{|l|l|l|l|}
\hline Ingredient (\%) & $\mathbf{( d ~ 1 - 1 4 )}$ & $\mathbf{( d ~ 1 5 - 2 8 )}$ & $\mathbf{( d ~ 2 9 - 4 2 )}$ \\
\hline Corn & 55.00 & 57.65 & 59.03 \\
\hline Soybean meal & 38.00 & 34.87 & 33.80 \\
\hline Soybean oil & 3.00 & 3.50 & 4.00 \\
\hline Dicalcium phosphate & 1.85 & 1.60 & 1.70 \\
\hline limestone & 1.17 & 1.20 & 1.38 \\
\hline salt & 0.35 & 0.35 & 0.30 \\
\hline Vitamin premix ${ }^{\mathrm{a}}$ & 0.03 & 0.03 & 0.02 \\
\hline Choline chloride (50\%) & 0.10 & 0.07 & 0.05 \\
\hline Mineral premix ${ }^{\mathrm{b}}$ & 0.20 & 0.20 & 0.20 \\
\hline DL-Met & 0.25 & 0.16 & 0.07 \\
\hline HCl-Lys & 0.05 & 0.04 & 0.03 \\
\hline total & 100 & 100 & 100 \\
\hline Calculated nutrition levels & \multicolumn{4}{|l}{} \\
\hline ME (MJ/Kg) & 12.16 & 12.78 & 12.82 \\
\hline CP (\%) & 20.74 & 19.60 & 18.00 \\
\hline Met+Cys (\%) & 0.92 & 1.05 & 0.64 \\
\hline Lys (\%) & 1.1 & 0.95 & 0.9 \\
\hline Ca (\%) & 1.08 & 0.95 & 0.9 \\
\hline P (\%) & 0.71 & 0.64 & 0.55 \\
\hline & \multicolumn{2}{|l}{} \\
\hline
\end{tabular}

Table 3. Dietary compositions and nutrient levels of broilers (as-fed basis). ${ }^{a}$ Vitamin premix containing the following content, per kilogram of diet: vitamin A, 10,000 IU; vitamin $\mathrm{D}_{3}$ (cholecalciferol), $3500 \mathrm{IU}$; vitamin $\mathrm{E}$ (DL- $\alpha$-tocopheryl acetate), $60 \mathrm{mg}$; vitamin $\mathrm{K}$ (menadione), $3 \mathrm{mg}$; thiamine, $3 \mathrm{mg}$; riboflavin, $6 \mathrm{mg}$; pyridoxine, $5 \mathrm{mg}$; vitamin $\mathrm{B}_{12}$ (cyanocobalamin), $0.01 \mathrm{mg}$; niacin, $45 \mathrm{mg}$; pantothenic acid (D-calcium pantothenate), $11 \mathrm{mg}$; folic acid, $1 \mathrm{mg}$; biotin, $0.15 \mathrm{mg}$; choline chloride, $500 \mathrm{mg}$; ethoxyquin (antioxidant), $150 \mathrm{mg}$. ${ }^{\mathrm{b}}$ Mineral premix containing the following content, per kilogram of diet: Fe, $60 \mathrm{mg}$; Mn, $100 \mathrm{mg}$; Zn, $60 \mathrm{mg}$; Cu, $10 \mathrm{mg}$; I, $1 \mathrm{mg}$; Co, $0.2 \mathrm{mg}$; Se, $0.15 \mathrm{mg}$.

\section{Materials and Methods}

PEO chemical composition. PEO product purchased from Guangdong Ruisheng Technology Co., Ltd (Guangzhou, China), which contained 65\% silicon dioxide and 30\% plant essential oils. The composition of plant essential oil was analysis by GC/MS (Agilent 5973 Network Mass Selective Detector, Agilent Technologies,USA), the relative content of the active components were $78.3 \%$ Cinnamic dehyde $(\mathrm{RT}=33.6)$, $4 \%$ Isophorone $(\mathrm{RT}=22.9)$, and eugenol (2.7\%) (Fig. 5).

Animals, treatment, management, and ethics statement. One-day-old male Cobb 500 chickens (234) were randomly divided into three groups, and each group was replicated six times with 12 chickens in each replicate. Each group was fed with: (1) a basal diet (CT), without any additives; (2) a basal diet with $400 \mathrm{mg} / \mathrm{kg}$ (PEO); (3) a basal diet with $30 \mathrm{mg} / \mathrm{kg}$ virginiamycin (VIRG). Virginiamycin purchased from Phibro Animal Health Corporation (USA). The corn-soybean-based basal diet was used, and the nutritional level of the diet was prepared following the NRC (1994) feeding standards. All experiments were performed in accordance with the approved guidelines and regulations. The diet composition and nutrient levels are shown in Table 3.

Chickens were reared in a coop $(100 \mathrm{~cm} \times 80 \mathrm{~cm})$ with two water nipples per pen. Chickens were raised routinely and immunized according to the normal immunization procedures. The diet was available ad libitum throughout the trial period. The temperature of the house was maintained at the optimum temperature of broiler chickens at $33-34^{\circ} \mathrm{C}$ for the first week, and decreased from the second week by $3^{\circ} \mathrm{C}$ per week until it reached $22^{\circ} \mathrm{C}$. All experimental protocols were approved by the China Agricultural University Animal Care Committee (permit number SYXK20171208).

Sampling collection. At 28 days of age, six chickens per treatment group (one chicken per repeat) were randomly selected to collected blood from the left brachial vein. After blood was collected, the chicken was injected intravenously pentobarbital sodium $(30 \mathrm{mg} / \mathrm{kg}$ body weight) and cervical dislocation was executed about $2 \mathrm{~g}$ of cecal digestive fluid was collected and placed in two sterilizing tubes, immediately frozen in liquid nitrogen and stored at $-80^{\circ} \mathrm{C}$. One sample was used for DNA extraction and Pyrosequencing, and second one for global metabolomic analysis. The blood samples were centrifuged for $15 \mathrm{~min}\left(1,500 \mathrm{~g}, 4^{\circ} \mathrm{C}\right)$. serum sample was stored at $-80^{\circ} \mathrm{C}$ for UPLC-Q-TOF/MS analysis.

DNA Extraction and 16S rDNA Amplicon Pyrosequencing. Total bacterial genomic DNA was extracted from each digested sample using the Fast DNA SPIN extraction kits (MP Biomedicals, Santa Ana, CA, USA). DNA extracts were stored at $-20^{\circ} \mathrm{C}$ prior to further analysis. Extracted DNAs were measured using a NanoDrop ND-1000 spectrophotometer (Thermo Fisher Scientific, Waltham, MA, USA) and agarose gel electrophoresis. 
PCR amplification was performed according to the method of Jinfeng Song ${ }^{59}$. The V3-V4 region of $16 \mathrm{~S}$ rRNA genes was amplified using the 338F-806R primer set (338F: 55'-ACTCCTACGGGAGGCAGCA-3'. 806R:5' GGACTACHVGGGTWTCTAAT-3'). The sample specificity 7-bp barcode was added to the primer for multiple sequencing. Thermal cycle included initial denaturation of $98^{\circ} \mathrm{C}$ for $2 \mathrm{~min}$, followed by 25 cycles consisting of denaturation of $98^{\circ} \mathrm{C}$ for $15 \mathrm{~s}$, annealing at $55^{\circ} \mathrm{C}$ for $30 \mathrm{~s}$, extension of $72^{\circ} \mathrm{C}$ for $30 \mathrm{~s}$, and final extension of $72^{\circ} \mathrm{C}$ for 5 min. PCR amplifiers were purified using Agencourt AMPure Beads (Beckman Coulter, Indianapolis, IN, USA) and quantified using PicoGreen dsDNA Assay Kit (Invitrogen, Carlsbad, CA, USA) ${ }^{59}$. After the individual sample was quantified, the amplicon were collected in equal amount, and pair-end $2 \times 300 \mathrm{bp}$ sequencing was performed using the Illlumina MiSeq platform with MiSeq Reagent Kit v3 at Shanghai Personal Biotechnology Co., Ltd (Shanghai, China) ${ }^{5}$.

Sequence analysis. The sequencing data were processed using the Quantitative Insights Into Microbial Ecology (QIIME, v1.8.0) pipeline, following Caporaso et al. $(2010)^{60}$. Briefly, raw sequencing reads with complete barcode matches were assigned to the appropriate sample and identified as valid. Sequences $<150 \mathrm{bp}$ long, with average Phred scores $<20$, containing ambiguous bases, or with mononucleotide repeats longer than 8 bp were considered low-quality and were excluded from further analysis ${ }^{61}$. Paired-end reads were assembled using $\mathrm{FLASH}^{62}$. After chimera detection, UCLUST ${ }^{63}$ was used to group the remaining high-quality sequences into OTUs based on a minimum sequence identity of $97 \%$. A representative sequence was identified for each OTU using default parameters. The OTUs were taxonomically classified using BLAST; each representative sequence was searched against the Greengenes Database ${ }^{64}$ and the best hit was selected ${ }^{65}$. An OTU table was used to record the abundance and taxonomic affiliation membership of every OTU in each sample. OTUs representing less than $0.001 \%$ of all of the sequences across all of the samples were ignored. To maintain a constant sequencing depth across all of the samples, an averaged, rounded, rarefied OTU table was generated by re-sampling an average of 100 OTU subsets at a minimum sequencing depth of $90 \%$. This averaged OTU table was used for all of the subsequent analyses. Bioinformatics analyses of the sequence data were primarily performed in QIIME and R. v3.2.0. Raw reads were denoised and then cleaned to remove chimeras and low-quality sequences. OTU-level alpha diversity indices, such as Shannon diversity index, Chaol richness estimate, and observed species richness were calculated based on the OTU table in QIIME, and OTU-level rank abundance curves were generated to compare the evenness and richness of OTUs among samples. Principal component analysis (PCA) was performed at the genus level ${ }^{13}$. LEfSe (Linear discriminant analysis effect size) was performed to detect differential abundant taxa across groups using the default parameters ${ }^{66}$. Operational taxonomic units were clustered with a $97 \%$ similarity threshold. Alpha diversity analysis included Shannon diversity index, Chaol richness estimate, and observed species richness. Principal component analysis (PCA) was performed at the genus level ${ }^{13}$. LEfSe (Linear discriminant analysis effect size) was performed to detect differential abundant taxa across groups using the default parameters $^{66}$

Sample preparation for LC-MS/MS analysis. The serum $(100 \mu \mathrm{L})$ was added to $400 \mu \mathrm{L}$ of ice-chilled methanol/acetonitrile $(1: 1, \mathrm{v} / \mathrm{v})$ and centrifuged for $20 \mathrm{~min}\left(14,000 \mathrm{~g}, 4^{\circ} \mathrm{C}\right)$ to remove the protein. The supernatant was dried in a vacuum centrifuge. For LC-MS analysis ${ }^{67}$, the samples were re-dissolved in $100 \mu \mathrm{L}$ of acetonitrile/water $(1: 1, \mathrm{v} / \mathrm{v})$. The same procedure was conducted using $100 \mathrm{mg}$ sample from frozen cecal contents. One milliliter of cold methanol/acetonitrile $(1: 1, \mathrm{v} / \mathrm{v})$ was added to each sample and mixed. The mixture was separated by centrifugation for $15 \mathrm{~min}\left(14,000 \mathrm{~g}, 4^{\circ} \mathrm{C}\right)$. The supernatant was dried under vacuum centrifuge. Through the vortex dissolved in $100 \mu \mathrm{L}$ acetonitrile and water $(1: 1, \mathrm{v} / \mathrm{v})$, and under the $49^{\circ} \mathrm{C}$ to $12,000 \mathrm{~g}$ centrifugal for $15 \mathrm{~min}$ utes. Monitor the stability and repeatability of the instrument analysis, quality control (QC) samples from the pool by $10 \mu \mathrm{L}$ per sample and analysis along with other samples. Every 6 samples were periodically inserted into QC samples for analysis ${ }^{68}$.

LC-MS/MS Analysis for serum and cecal contents. Determination of cecal contents and serum by using a UHPLC (1290 Infinity LC, Agilent Technologies) coupled to a quadrupole time-of-flight (AB Sciex TripleTOF 6600) in Shanghai Applied Protein Technology Co., Ltd. According to Wenqiang Fan's method, the HILIC separation was carried out samples were analyzed by using a $2.1 \mathrm{~mm} \times 100 \mathrm{~mm}$ ACQUIY UPLC BEH $1.7 \mu \mathrm{m}$ column (waters, Ireland) ${ }^{69}$. In the ESI positive and negative mode, the mobile phase contained $\mathrm{A}=25 \mathrm{mM}$ ammonium acetate and $25 \mathrm{mM}$ aqueous ammonium hydroxide, $\mathrm{B}=$ acetonitrile. The gradient was $85 \% \mathrm{~B}$ for 1 minute, followed by a linear drop to $65 \%$ in 11 minutes, then a drop to $40 \%$ in 0.1 minutes for 4 minutes, and ultimately increased to $85 \%$ in 0.1 minutes for 5 minutes with a rebalancing period.

ESI conditions were set as follows: the ion source Gas1 (Gas1) was 60, the ion source Gas2 (Gas2) was 60, the curtain gas (CUR) was 30, the source temperature was $600^{\circ} \mathrm{C}$, and the ion spray voltage fluctuation (ISVF) was $\pm 5500 \mathrm{~V}$. In the MS only acquisition, the instrument was set to collect data in the range of 60-1000 Da, and the TOF MS scanning accumulation time was set to $0.20 \mathrm{~s} / \mathrm{spectrum}$. In the automatic MS/MS acquisition, the instrument was set at the range of $\mathrm{m} / \mathrm{z}$ and above $25-1000 \mathrm{Da}$, and the production scanning accumulation time was set at $0.05 \mathrm{~s} /$ spectrum $^{70}$. The production scan is acquired using information dependent acquisition (IDA) with the selection of high sensitivity mode. The parameters were set as follows ${ }^{71}$ : the collision energy (CE) was fixed at $35 \mathrm{~V}$ with $\pm 15 \mathrm{eV}$; declustering potential (DP), $60 \mathrm{~V}(+)$ and $-60 \mathrm{~V}(-)$; exclude isotopes within $4 \mathrm{Da}$; and 10 candidate ions to monitor per cycle.

The raw MS data (wiff. scan files) were converted to MzXML files using ProteoWizard MSConvert before importing it into freely available XCMS software ${ }^{67}$. For peak selection, the following parameters were used: centWave $\mathrm{m} / \mathrm{z}=25 \mathrm{ppm}$, peak width $=\mathrm{c}(10,60)$, prefilter $=\mathrm{c}(10,100)$. For peak grouping, $\mathrm{bw}=5, \operatorname{mzwid}=0.025$, $\operatorname{minfrac}=0.5$ were used $^{71}$. CAMERA (Collection of Algorithms of MEtabolite pRofile Annotation) was used for annotation of isotopes and admixtures. In the extracted ion features, only more than $50 \%$ of the variables of at 
least one set of non-zero measurements were retained. The variation coefficient $(\mathrm{CV})$ of metabolites in the QC samples was set at a threshold value of $30 \%$, which was used as the standard for evaluating the repeatability of the metabolomics data set. Compound identification of metabolites was performed by comparing the accuracy of $\mathrm{m} / \mathrm{z}$ value $(<25 \mathrm{ppm})$, and MS/MS spectra with an in-house database established using reliable standards ${ }^{72}$.

Statistical analysis. After normalizing the processed data to the total peak intensity, it was uploaded before importing into SIMCA-P (version 14.1, Umetrics, Umea, Sweden), for multivariate data analysis, including pareto scale principal component analysis (PCA) and orthogonal partial least squares discriminant analysis $(\text { OPLS-DA })^{71}$. The importance of each variable in the OPLS-DA model in the projection (VIP) value was calculated to indicate its contribution to the classification. Metabolites with the VIP score $>1$ were further applied to Student's t-test at univariate level to measure the significance of each metabolite, the $\mathrm{P}$ values less than 0.05 were considered as statistically significant. The correlative analysis between different cecal microbial species and microbial or serum metabolites was used for calculating by Spearman's correlations coefficient ${ }^{13}$.

Received: 29 August 2019; Accepted: 6 February 2020;

Published online: 25 March 2020

\section{References}

1. Apajalahti, J. Comparative gut microflora, metabolic challenges, and potential opportunities. J APPL POULTRY RES 14, 444-453 (2005).

2. Turnbaugh, P. J. et al. An obesity-associated gut microbiome with increased capacity for energy harvest. NATURE 444, 1027-1031, https://doi.org/10.1038/nature05414 (2006).

3. Wei, S., Morrison, M. \& Yu, Z. Bacterial census of poultry intestinal microbiome. POULTRY SCI 92, 671-683, https://doi. org/10.3382/ps.2012-02822 (2013).

4. Li, M. et al. Symbiotic gut microbes modulate human metabolic phenotypes. P NATL A SCI INDIA A 105, 2117-2122 (2008).

5. Xu, J. et al. Metabolomic Analysis and Mode of Action of Metabolites of Tea Tree Oil Involved in the Suppression of Botrytis cinerea. Front Microbiol 8, https://doi.org/10.3389/fmicb.2017.01017 (2017).

6. Marchesi, J. R. et al. The gut microbiota and host health: a new clinical frontier. Gut 65, 330-339 (2016).

7. Prakash, U. N. \& Srinivasan, K. Beneficial influence of dietary spices on the ultrastructure and fluidity of the intestinal brush border in rats. BRIT J NUTR 104, 31-39 (2010).

8. Knarreborg, A., Lauridsen, C., Engberg, R. M. \& Jensen, S. K. Dietary antibiotic growth promoters enhance the bioavailability of $\alpha$-tocopheryl acetate in broilers by altering lipid absorption. J NUTR 134, 1487-1492, https://doi.org/10.1093/jn/134.6.1487 (2004).

9. Han, G. G. et al. Relationship between the microbiota in different sections of the gastrointestinal tract, and the body weight of broiler chickens. SpringerPlus 5, 911, https://doi.org/10.1186/s40064-016-2604-8 (2016).

10. Gadde, U. D., Oh, S., Lillehoj, H. S. \& Lillehoj, E. P. Antibiotic growth promoters virginiamycin and bacitracin methylene disalicylate alter the chicken intestinal metabolome. SCI REP-UK 8, 3592, https://doi.org/10.1038/s41598-018-22004-6 (2018).

11. Gadde, U., Kim, W., Oh, S. \& Lillehoj, H. S. Alternatives to antibiotics for maximizing growth performance and feed efficiency in poultry: a review. ANIM HEALTH RES REV 18, 26-45 (2017).

12. Brenes, A. \& Roura, E. Essential oils in poultry nutrition: Main effects and modes of action. ANIM FEED SCI TECH 158, 1-14 (2010).

13. Li, Y. et al. Intestinal Microbiome-Metabolome Responses to Essential Oils in Piglets. Front Microbiol 9, 1988-1988, https://doi. org/10.3389/fmicb.2018.01988 (2018).

14. Zeng, Z., Zhang, S., Wang, H. \& Piao, X. Essential oil and aromatic plants as feed additives in non-ruminant nutrition: a review. $J$ ANIM SCI BIOTECHNO 6, 7 (2015).

15. Ran, C. et al. Thymol and carvacrol affect hybrid tilapia through the combination of direct stimulation and an intestinal microbiotamediated effect: insights from a germ-free zebrafish model. JNUTR 146, 1132-1140 (2016).

16. Di Pasqua, R. et al. Membrane toxicity of antimicrobial compounds from essential oils. J AGR FOOD CHEM 55, 4863-4870 (2007).

17. Cho, J. H., Kim, H. J. \& Kim, I. H. Effects of phytogenic feed additive on growth performance, digestibility, blood metabolites, intestinal microbiota, meat color and relative organ weight after oral challenge with Clostridium perfringens in broilers. LIVEST SCI 160, 82-88 (2014).

18. Huang, P. et al. The chicken gut metagenome and the modulatory effects of plant-derived benzylisoquinoline alkaloids. MICROBIOME 6, 211, https://doi.org/10.1186/s40168-018-0590-5 (2018).

19. Giannenas, I. et al. Dietary supplementation of benzoic acid and essential oil compounds affects buffering capacity of the feeds, performance of turkey poults and their antioxidant status, $\mathrm{pH}$ in the digestive tract, intestinal microbiota and morphology. ASIAN AUSTRAL J ANIM 27, 225 (2014).

20. Altop, A., Erener, G., Duru, M. E. \& Isik, K. Effects of essential oils from Liquidambar orientalis Mill. leaves on growth performance, carcass and some organ traits, some blood metabolites and intestinal microbiota in broilers. British Poultry Science 59, 121-127, https://doi.org/10.1080/00071668.2017.1400657 (2018).

21. Diaz Carrasco, J. M. et al. Use of Plant Extracts as an Effective Manner to Control Clostridium perfringens Induced Necrotic Enteritis in Poultry. Biomed Res Int 2016, 3278359-3278359, https://doi.org/10.1155/2016/3278359 (2016).

22. Zhu, N. et al. Modulation of Growth Performance and Intestinal Microbiota in Chickens Fed Plant Extracts or Virginiamycin. FRONT MICROBIOL 10 (2019).

23. Windisch, W., Schedle, K., Plitzner, C. \& Kroismayr, A. Use of phytogenic products as feed additives for swine and poultry1. Journal of Animal Science 86, E140-E148, https://doi.org/10.2527/jas.2007-0459 (2008).

24. Diaz-Sanchez, S., D’Souza, D., Biswas, D. \& Hanning, I. J. P. S. Botanical alternatives to antibiotics for use in organic poultry production. 94, 1419-1430 (2015).

25. Lee, K. W. et al. Effects of dietary essential oil components on growth performance, digestive enzymes and lipid metabolism in female broiler chickens. BRIT POULTRY SCI 44, 450-457 (2003).

26. Jang, I. S. et al. Influence of essential oil components on growth performance and the functional activity of the pancreas and small intestine in broiler chickens. ASIAN AUSTRAL J ANIM 17, 394-400 (2004).

27. Hashemi, S. R. \& Davoodi, H. Herbal plants and their derivatives as growth and health promoters in animal nutrition. VET RES COMMUN 35, 169-180(2011).

28. Thapa, D., Losa, R., Zweifel, B. \& Wallace, R. J. Sensitivity of pathogenic and commensal bacteria from the human colon to essential oils. MICROBIOLOGY 158, 2870-2877 (2012).

29. Placha, I. et al. Effect of thyme essential oil and selenium on intestine integrity and antioxidant status of broilers. BRIT POULTRY SCI 55, 105-114 (2014).

30. Torok, V. A., Allison, G. E., Percy, N. J., Ophel-Keller, K. \& Hughes, R. J. Influence of antimicrobial feed additives on broiler commensal posthatch gut microbiota development and performance. Appl. Environ. Microbiol. 77, 3380-3390, https://doi. org/10.1128/AEM.02300-10 (2011). 
31. Abe, K. et al. Anaerocella delicata gen. nov., sp. nov., a strictly anaerobic bacterium in the phylum Bacteroidetes isolated from a methanogenic reactor of cattle farms. APPL. MICROBIOL. 58, 405-412 (2012).

32. Eija Könönen, S. Y. et al. Bergey’s Manual of Systematics of Archaea and Bacteria(Alistipes)(American Cancer Society, 2015).

33. Graf, J. J. T. P. O. M. L. O. B. \& Archaea, T. The family rikenellaceae. 857-859 (2014)

34. Neyrinck, A. M. et al. Dietary modulation of clostridial cluster XIVa gut bacteria (Roseburia spp.) by chitin-glucan fiber improves host metabolic alterations induced by high-fat diet in mice. The Journal of Nutritional Biochemistry 23, 51-59, https://doi. org/10.1016/j.jnutbio.2010.10.008 (2012).

35. Du, T. et al. Metabolic profiling of femoral muscle from rats at different periods of time after death. PLoS One 13, e0203920, https:// doi.org/10.1371/journal.pone.0203920 (2018)

36. Huang, M. K. et al. Effects of Lactobacilli and an acidophilic fungus on the production performance and immune responses in broiler chickens. POULTRY SCI 83, 788-795, https://doi.org/10.1093/ps/83.5.788 (2004).

37. De Boever, P. et al. Protective effect of the bile salt hydrolase-active Lactobacillus reuteri against bile salt cytotoxicity. APPL MICROBIOL BIOT 53, 709-714 (2000).

38. Stanley, D. et al. Intestinal microbiota associated with differential feed conversion efficiency in chickens. APPL MICROBIOL BIOT 96, 1361-1369 (2012)

39. Fåk, F. \& Bäckhed, F. Lactobacillus reuteri prevents diet-induced obesity, but not atherosclerosis, in a strain dependent fashion in Apoe-/- mice. PLoS One 7, e46837 (2012).

40. Stanley, D., Hughes, R. J., Geier, M. S. \& Moore, R. J. Bacteria within the gastrointestinal tract microbiota correlated with improved growth and feed conversion: challenges presented for the identification of performance enhancing probiotic bacteria. Front Microbiol 7, 187 (2016).

41. Nie, C. et al. Cross-sectional and dynamic change of serum metabolite profiling for $\mathrm{H}$ epatitis B-related acute-on-chronic liver failure by UPLC/MS. J VIRAL HEPATITIS 21, 53-63 (2014).

42. Quinn, M. T., Parthasarathy, S. \& Steinberg, D. Lysophosphatidylcholine: a chemotactic factor for human monocytes and its potential role in atherogenesis. Proc Natl Acad Sci USA 85, 2805-2809, https://doi.org/10.1073/pnas.85.8.2805 (1988).

43. Rytczak, P., Koziołkiewicz, M. \& Okruszek, A. J. N. J. O. C. The chemical synthesis of phosphorothioate and phosphorodithioate analogues of lysophosphatidic acid (LPA) and cyclic phosphatidic acid (CPA). NEW J CHEM 34, 1008-1017 (2010).

44. Jones, P. M. \& George, A. M. The ABC transporter structure and mechanism: perspectives on recent research. Cellular and Molecular Life Sciences CMLS 61, 682-699, https://doi.org/10.1007/s00018-003-3336-9 (2004).

45. Feighner, S. D. \& Dashkevicz, M. P. J. A. E. M. Subtherapeutic levels of antibiotics in poultry feeds and their effects on weight gain, feed efficiency, and bacterial cholyltaurine hydrolase activity. 53, 331-336 (1987).

46. Knarreborg, A., Lauridsen, C., Engberg, R. M. \& Jensen, S. K. Dietary Antibiotic Growth Promoters Enhance the Bioavailability of $\alpha$-Tocopheryl Acetate in Broilers by Altering Lipid Absorption. The Journal of Nutrition 134, 1487-1492, https://doi.org/10.1093/ jn/134.6.1487 (2004).

47. Danzeisen, J. L., Kim, H. B., Isaacson, R. E., Tu, Z. J. \& Johnson, T. J. Modulations of the chicken cecal microbiome and metagenome in response to anticoccidial and growth promoter treatment. PLoS One 6, e27949 (2011).

48. Lan, Y., Verstegen, M., Tamminga, S. \& Williams, B. The role of the commensal gut microbial community in broiler chickens. WORLD POULTRY SCI J 61, 95-104 (2005).

49. Guban, J., Korver, D. R., Allison, G. E. \& Tannock, G. W. Relationship of dietary antimicrobial drug administration with broiler performance, decreased population levels of Lactobacillus salivarius, and reduced bile salt deconjugation in the ileum of broiler chickens. POULTRY SCI 85, 2186-2194 (2006).

50. Yu, M. et al. Marked Response in Microbial Community and Metabolism in the Ileum and Cecum of Suckling Piglets After Early Antibiotics Exposure. Front Microbiol 9, 1166, https://doi.org/10.3389/fmicb.2018.01166 (2018).

51. Gong, J. et al. Effects of zinc bacitracin, bird age and access to range on bacterial microbiota in the ileum and caeca of broiler chickens. J APPL MICROBIOL 104, 1372-1382 (2008).

52. Costa, M. C. et al. Different antibiotic growth promoters induce specific changes in the cecal microbiota membership of broiler chicken. PLoS One 12, e0171642 (2017).

53. Neumann, A. \& Suen, G. Differences in major bacterial populations in the intestines of mature broilers after feeding virginiamycin or bacitracin methylene disalicylate. J APPL MICROBIOL 119, 1515-1526 (2015).

54. Whelan, J. \& Fritsche, K. Linoleic acid. Adv Nutr 4, 311-312, https://doi.org/10.3945/an.113.003772 (2013).

55. Breyer, M. D. \& Breyer, R. M. G Protein-Coupled Prostanoid Receptors and the Kidney. Annual Review of Physiology 63, 579-605, https://doi.org/10.1146/annurev.physiol.63.1.579 (2001).

56. Hata, A. N. \& Breyer, R. M. Pharmacology and signaling of prostaglandin receptors: Multiple roles in inflammation and immune modulation. PHARMACOL THERAPEUT 103, 147-166, https://doi.org/10.1016/j.pharmthera.2004.06.003 (2004).

57. Zhou, S.-S. \& Zhou, Y. J. W. J. O. D. Excess vitamin intake: An unrecognized risk factor for obesity. 5, 1 (2014).

58. Moreno-Navarrete, J. M. et al. Metabolomics uncovers the role of adipose tissue PDXK in adipogenesis and systemic insulin sensitivity. 59, 822-832 (2016).

59. Song, J. et al. Effects of Graphene on Bacterial Community Diversity and Soil Environments of Haplic Cambisols in Northeast China. FORESTS 9, 677, https://doi.org/10.3390/f9110677 (2018).

60. Caporaso, J. G. et al. QIIME allows analysis of high-throughput community sequencing data. NAT METHODS 7, 335 (2010).

61. Gill, S. R. et al. Metagenomic analysis of the human distal gut microbiome. Science 312, 1355-1359 (2006).

62. Magoč, T. \& Salzberg, S. L. J. B. FLASH: fast length adjustment of short reads to improve genome assemblies. BIOINFORMATICS1 27, 2957-2963 (2011)

63. Edgar, R. C. J. B. Search and clustering orders of magnitude faster than BLAST. BIOINFORMATICS1 26, 2460-2461 (2010).

64. DeSantis, T. Z. et al. Greengenes, a chimera-checked $16 \mathrm{~S}$ rRNA gene database and workbench compatible with ARB. APPL ENVIRON MICROB 72, 5069-5072 (2006).

65. Altschul, S. F. et al. Gapped BLAST and PSI-BLAST: a new generation of protein database search programs. NUCLEIC ACIDS RES 25, 3389-3402 (1997)

66. Zhao, T., Shen, X., Dai, C. \& Cui, L. Benefits of procyanidins on gut microbiota in Bama minipigs and implications in replacing antibiotics. J VET SCI 19, 798-807 (2018).

67. Benton, H. P. et al. Autonomous Metabolomics for Rapid Metabolite Identification in Global Profiling. Analytical Chemistry 87, 884-891, https://doi.org/10.1021/ac5025649 (2015).

68. Zhou, X. et al. Polyunsaturated fatty acids metabolism, purine metabolism and inosine as potential independent diagnostic biomarkers for major depressive disorder in children and adolescents. MOL PSYCHIATR, 1 (2018).

69. Fan, W. et al. Proteomics integrated with metabolomics: analysis of the internal causes of nutrient changes in alfalfa at different growth stages. BMC plant biology 18, 78 (2018).

70. Zhao, G. et al. Metabolomics analysis of alloxan-induced diabetes in mice using UPLC-Q-TOF-MS after Crassostrea gigas polysaccharide treatment. INT J BIOL MACROMOL 108, 550-557 (2018).

71. Zhao, Y. et al. UPLC-QTOF/MS-based metabolomics analysis of plasma reveals an effect of Xue-Fu-Zhu-Yu capsules on blood-stasis syndrome in CHD rats. J ETHNOPHARMACOL 241, 111908, https://doi.org/10.1016/j.jep.2019.111908 (2019).

72. Xu, J. et al. Rapid degradation of long-chain crude oil in soil by indigenous bacteria using fermented food waste supernatant. WASTE MANAGE 85, 361-373 (2019). 


\section{Acknowledgements}

This work was supported by National Natural Science Foundation of China (Project No. 3146068; Beijing, P. R. China).

\section{Author contributions}

Y.C. and N.Z. designed the experiments and wrote the paper; J.W., T.X. and Y.C. performed the experiments and collected the samples. Y.C., J.W. and L.Y. analyzed the data. J.W., Y.C. and N.Z. were responsible for the final content. All authors have read and approved the final manuscript.

\section{Competing interests}

The authors declare no competing interests.

\section{Additional information}

Supplementary information is available for this paper at https://doi.org/10.1038/s41598-020-60135-x.

Correspondence and requests for materials should be addressed to N.Z.

Reprints and permissions information is available at www.nature.com/reprints.

Publisher's note Springer Nature remains neutral with regard to jurisdictional claims in published maps and institutional affiliations.

(c) (i) Open Access This article is licensed under a Creative Commons Attribution 4.0 International License, which permits use, sharing, adaptation, distribution and reproduction in any medium or format, as long as you give appropriate credit to the original author(s) and the source, provide a link to the Creative Commons licence, and indicate if changes were made. The images or other third party material in this article are included in the article's Creative Commons licence, unless indicated otherwise in a credit line to the material. If material is not included in the article's Creative Commons licence and your intended use is not permitted by statutory regulation or exceeds the permitted use, you will need to obtain permission directly from the copyright holder. To view a copy of this licence, visit http://creativecommons.org/licenses/by/4.0/.

(C) The Author(s) 2020 